\title{
Fair Blind Signatures without Random Oracles
}

\author{
Georg Fuchsbauer and Damien Vergnaud \\ École normale supérieure, LIENS - CNRS - INRIA, Paris, France \\ http://www.di.ens.fr/\{ ${ }^{\text {fuchsbau, }}$, vergnaud $\}$
}

\begin{abstract}
A fair blind signature is a blind signature with revocable anonymity and unlinkability, i.e., an authority can link an issuing session to the resulting signature and trace a signature to the user who requested it. In this paper we first revisit the security model for fair blind signatures given by Hufschmitt and Traoré in 2007. We then give the first practical fair blind signature scheme with a security proof in the standard model. Our scheme satisfies a stronger variant of the Hufschmitt-Traoré model.

Keywords. Blind signatures, Revocable anonymity, Standard model, Groth-Sahai proof system.
\end{abstract}

\section{Introduction}

A blind signature scheme is a protocol for obtaining a signature from an issuer such that the issuer's view of the protocol cannot be linked to the resulting message/signature pair. Blind signatures are employed in privacy-related protocols where the issuer and the message author are different parties (e.g., e-voting or e-cash systems). However, blind signature schemes provide perfect unlinkability and could therefore be misused by dishonest users. Fair blind signatures were introduced by Stadler, Piveteau and Camenisch [SPC95] to prevent abuse of unlinkability. They allow two types of blindness revocation: linking a signature to the user who asked for the signature and identifying a signature that resulted from a given signing session. A security model for fair blind signatures was introduced by Hufschmitt and Traoré [HT07].

We first revisit their security model and give a stronger variant. We then present the first efficient fair blind signature scheme with a standard-model security proof (i.e., without resorting to the random oracle heuristic) in the strengthened security model. We make extensive use of the non-interactive proof system due to Groth and Sahai [GS08] and of the automorphic signatures recently introduced by Fuchsbauer [Fuc09] and do not use interactive assumptions.

\subsection{Prior work}

The concept of blind signatures was introduced by Chaum in [Cha83]. A blind signature scheme is a cryptographic primitive that allows a user to obtain from the issuer (signer) a digital signature on a message of the user's choice in such a way that the issuer's view of the protocol cannot be linked to the resulting message/signature pair. 
Blind signatures have numerous applications including e-cash: they prevent linking the withdrawal of money and the payment made by the same customer. However, the impossibility to link withdrawals and payments might lead to frauds (money laundering, blackmailing, ...). Some applications therefore require means to identify the resulting signature from the transcript of a signatureissuing protocol or to link a message/signature pair to the corresponding signing session.

Fair blind signatures were introduced by Stadler, Piveteau and Camenisch in [SPC95] to provide these means. Several fair blind signature schemes have been proposed since then [SPC95,AO01,HT07] with applications to e-cash [GT03] or e-voting [CGT06]. In [HT07], Hufschmitt and Traoré presented a formal security model for fair blind signatures and a scheme based on bilinear maps that satisfies it in the random oracle model under an interactive assumption. In a recent independent work, Rückert and Schröder [RS10] proposed a generic construction of fair partially blind signatures [AF96].

\subsection{Our contribution}

As a first contribution, we strengthen the security model proposed in [HT07]. In our model, the algorithm opening a transcript not only returns information to identify the signature that resulted from it, but additionally outputs the user that requested the signature and gives a proof of correct tracing.

We give a definition of blindness analogously to [Oka06], but additionally provide tracing oracles to the adversary. We propose a traceability notion that implies the original one. Finally, we formalize the non-frameability notions analogously to [BSZ05], where it is the adversary's task to output a framing signature (or transcript) and a proof. We believe that our version of signature non-frameability is more intuitive: no corrupt issuer can output a transcript, a "framing" opening of it and a proof (in [HT07], the adversary must output a message/signature pair such that an honest transcript opens to it). (cf. $\S 2.3$ for details.)

In 2008, Groth and Sahai [GS08] proposed a way to produce efficient noninteractive zero-knowledge (NIZK) and non-interactive witness-indistinguishable (NIWI) proofs for (algebraic) statements related to groups equipped with a bilinear map. In particular, they give proofs of satisfiability of pairing-product equations ( $c f . \S 4.2$ ). In [Fuc09], Fuchsbauer introduced the notion of automorphic signatures whose verification keys lie in the message space, messages and signatures consist of group elements only, and verification is done by evaluating a set of pairing-product equations ( $c f . \S 5)$. Among several applications, he constructed an (automorphic) blind signature in the following way: the user commits to the message, and gives the issuer a randomized message; the issuer produces a "pre-signature" from which the user takes away the randomness to recover a signature. The actual signature is then a Groth-Sahai proof of knowledge of a signature, which guarantees unlinkability to the issuing.

In this paper, we modify Fuchsbauer's blind signature scheme in order to construct the first practical fair blind signature scheme with a security reduc- 
tion in the standard model. Our security analysis does not introduce any new computational assumptions and relies only on falsifiable assumptions [Nao03] $(c f . \S 3)$. First, we extend Fuchsbauer's automorphic signature so it can sign three messages at once. Then, since in fair blind signature schemes blindness has to hold even against adversaries provided with tracing oracles, we use Groth's technique from [Gro07] to achieve CCA-anonymous group signatures: instead of just committing to the tracing information, we additionally encrypt it (using Kiltz' tag-based encryption scheme [Kil06]) and provide NIZK proofs of consistency with the commitments. In order to achieve the strengthened notion of non-frameability, we construct simulation-sound NIZK proofs of knowledge of a Diffie-Hellman solution which consist of group elements only and are verified by checking a set of pairing-product equations (i.e. Groth-Sahai compatible proofs).

Since messages and signatures consist of group elements only and their verification is done by evaluating a set of pairing-product equations, our fair blind signatures are Groth-Sahai compatible themselves which makes them perfectly suitable to design efficient fair e-cash systems following the approach proposed in [GT03]. In addition, our scheme is compatible with the "generic" variant ${ }^{1}$ of Votopia $\left[\mathrm{OMA}^{+} 99\right]$ proposed by Canard, Gaud and Traoré in [CGT06]. Combined with a suitable mix-net (e.g. [GL07]), it provides a practical electronic voting protocol in the standard model including public verifiability, and compares favorably with other similar systems in terms of computational cost.

\section{The Model}

\subsection{Syntax}

Definition 1. A fair blind signature scheme is a 10-tuple

$$
\text { (Setup, IKGen, UKGen, Sign, User, Ver, TrSig, Trld, ChkSig, Chkld) }
$$

of (interactive) (probabilistic) polynomial-time Turing machines ((P)PTs):

Setup is a PPT that takes as input an integer $\lambda$ and outputs the parameters pp and the revocation key rk. We call $\lambda$ the security parameter.

IKGen is a PPT that takes as input the parameters pp and outputs a pair (ipk, isk), the issuer's public and secret key.

UKGen is a PPT that takes as input the parameters pp and outputs a pair (upk, usk), the user's public and secret key.

Sign and User are interactive PPTs such that User takes as inputs the parameters pp, the issuer's public key ipk, the user's secret key usk and a bit string $m$; Sign takes as input pp, the issuer's secret key isk and user public key upk. Sign and User engage in the signature issuing protocol and when they stop, Sign outputs completed or not-completed while User outputs $\perp$ or a bit string $\sigma$.

\footnotetext{
${ }^{1}$ This variant was used during the French referendum on the European Constitution in May 2005.
} 
Ver is a deterministic PT that on input the parameters pp, an issuer public key ipk and a pair of bit strings $(m, \sigma)$ outputs either 0 or 1 . If it outputs 1 then $\sigma$ is a valid signature on the message $m$

TrSig is a deterministic PT that on input pp, an issuer public key ipk, a transcript of a signature issuing protocol and a revocation key rk outputs three bit strings (upk, $\left.i d_{\sigma}, \pi\right)$.

Trld is a deterministic PT that on input pp, an issuer public key ipk, a pair message/signature $(m, \sigma)$ for ipk and a revocation key rk outputs two bit strings (upk, $\pi$ ).

ChkSig is a deterministic PT that on input pp, an issuer public key ipk, a transcript of a signature issuing protocol, a pair message/signature $(m, \sigma)$ for ipk and three bit strings (upk, id $\left.{ }_{\sigma}, \pi\right)$, outputs either 0 or 1.

Chkld is a deterministic PT that on input pp, an issuer public key ipk, a pair message/signature $(m, \sigma)$ for ipk and two bit strings (upk, $\pi$ ), outputs either 0 or 1 .

For all $\lambda \in \mathbb{N}$, all pairs (pp, rk) output by $\operatorname{Setup}(\lambda)$ all pairs (ipk, isk) output by IKGen (pp), and all pairs (upk, usk) output by UKGen $(p p)$ :

1. if Sign and User follow the signature issuing protocol with input (pp, isk, upk) and (pp, usk, ipk, $m$ ) respectively, then Sign outputs completed and User outputs a bit string $\sigma$ that satisfies $\operatorname{Ver}(i p k,(m, \sigma))=1$;

2. on input ipk, the transcript trans of the protocol and rk, TrSig outputs three bit strings $\left(u p k, i_{\sigma}, \pi\right)$ s.t. ChkSig $\left(p p, i p k\right.$, trans, $\left.(m, \sigma),\left(u_{p k}, i d_{\sigma}, \pi\right)\right)=1$;

3. on input ipk, the pair $(m, \sigma)$ and rk, Trld outputs two bit strings (upk, $\pi$ ) such that $\operatorname{Chkld}(p p, i p k,(m, \sigma),(u p k, \pi))=1$.

\subsection{Security Definitions}

To define the security notions for fair blind signatures, we use a notation similar to the one in [BSZ05] used in [HT07]:

$H U$ denotes the set of honest users and $C U$ is the set of corrupted users.

AddU is an add-user oracle. By calling this oracle, the adversary creates a new user with keys (upk, usk). The oracle adds upk to $H U$ and returns it to the adversary.

CrptU is a corrupt-user oracle. The adversary calls this oracle with a pair (upk, usk) and upk added to the set $C U$.

USK is a user-secret-key oracle enabling the adversary to obtain the private key usk for some upk $\in H U$. The oracle transfers upk to $C U$ and returns usk.

User is an honest-user oracle. The adversary impersonating a corrupt issuer calls it with $(u p k, m)$. If upk $\in H U$, the experiment simulates the honest user holding upk running the signature issuing protocol with the adversary for message $m$. If the issuing protocol completed successfully, the adversary is given the resulting signature. The experiment keeps a list Set with entries of the form (upk, $m$, trans, $\sigma$ ), to record an execution of User, where trans is the transcript of the issuing protocol and $\sigma$ is the resulting signature. (Note that only valid $\sigma$ 's (i.e., the protocol was successful) are written to Set. 
Sign is a signing oracle. The adversary impersonating a corrupt user can use it to run the signature issuing protocol with the honest issuer. The experiment keeps a list Trans in which the transcripts trans ${ }_{i}$ resulting from Sign calls are stored.

Challenge $_{b}$ is a challenge oracle, which (w.l.o.g.) can only be called once. The adversary provides two user public keys $u p k_{0}$ and $u p k_{1}$ and two messages $m_{0}$ and $m_{1}$. The oracle first simulates User on inputs ( $\left.p p, i p k, u s k_{b}, m_{b}\right)$ and then, in a second protocol run, simulates User on inputs ( $\left.p p, i p k, u s k_{1-b}, m_{1-b}\right)$. Finally, the oracle returns $\left(\sigma_{0}, \sigma_{1}\right)$, the resulting signatures on $m_{0}$ and $m_{1}$.

TrSig (resp. Trld) is a signature (resp. identity) tracing oracle. When queried on the transcripts (or messages) emanating from a Challenge call, they return $\perp$.

Figure 1 formalizes the experiments for the following security notions:

Blindness. Not even the issuer with access to tracing oracles can link a message/signature pair to the signature issuing session it stems from.

Identity Traceability. No coalition of users can produce a set of signatures containing signatures which cannot be linked to an identity.

Signature Traceability. No one should be able to produce a message/signature pair which is not traced by any issuing transcript or two pairs which are traced by the same transcript.

Identity Non-Frameability. No coalition of issuer, users and tracing authority should be able to provide a signature and a proof that the signature opens to an honest user who did not ask for the signature.

Signature Non-Frameability. No coalition of issuer, users and tracing authority should be able to provide a transcript that either wrongfully opens to an honest signature or an honest user.

We say that a fair blind signature achieves blindness if for all PPT adversaries $\mathcal{A}$, the following is negligible: $\mid \operatorname{Pr}\left[\mathbf{E x p}_{\mathcal{A}}^{\text {blind-1 }}=1\right]-\operatorname{Pr}\left[\operatorname{Exp}_{\mathcal{A}}^{\text {blind-0 }}=1\right]-\frac{1}{2}$. The remaining security notions are achieved if for all $\mathrm{PPT} \mathcal{A}$, the probability that the corresponding experiment returns 1 is negligible.

\subsection{A Note on the Hufschmitt-Traoré Security Notions}

Blindness. In [HT07], the challenge oracle (called "Choose") is defined as follows: the adversary provides two user public keys $u p k_{0}$ and $u p k_{1}$ and a message, and obtains a signature under $u p k_{b}$. This gives a weak security guarantee, as the adversary - who impersonates the issuer - cannot actively participate in the issuing of the challenge signature. We define our oracle in the spirit of [Oka06]: the adversary impersonating the issuer chooses two users (and messages) which interact with him in random order; he gets to see both resulting signatures and has to determine the order of issuing.

Traceability Notions. Intuitively, identity traceability means that no coalition of users and the authority can create a message signature pair that is not traceable to a user, which is what was formalized in [HT07]. 


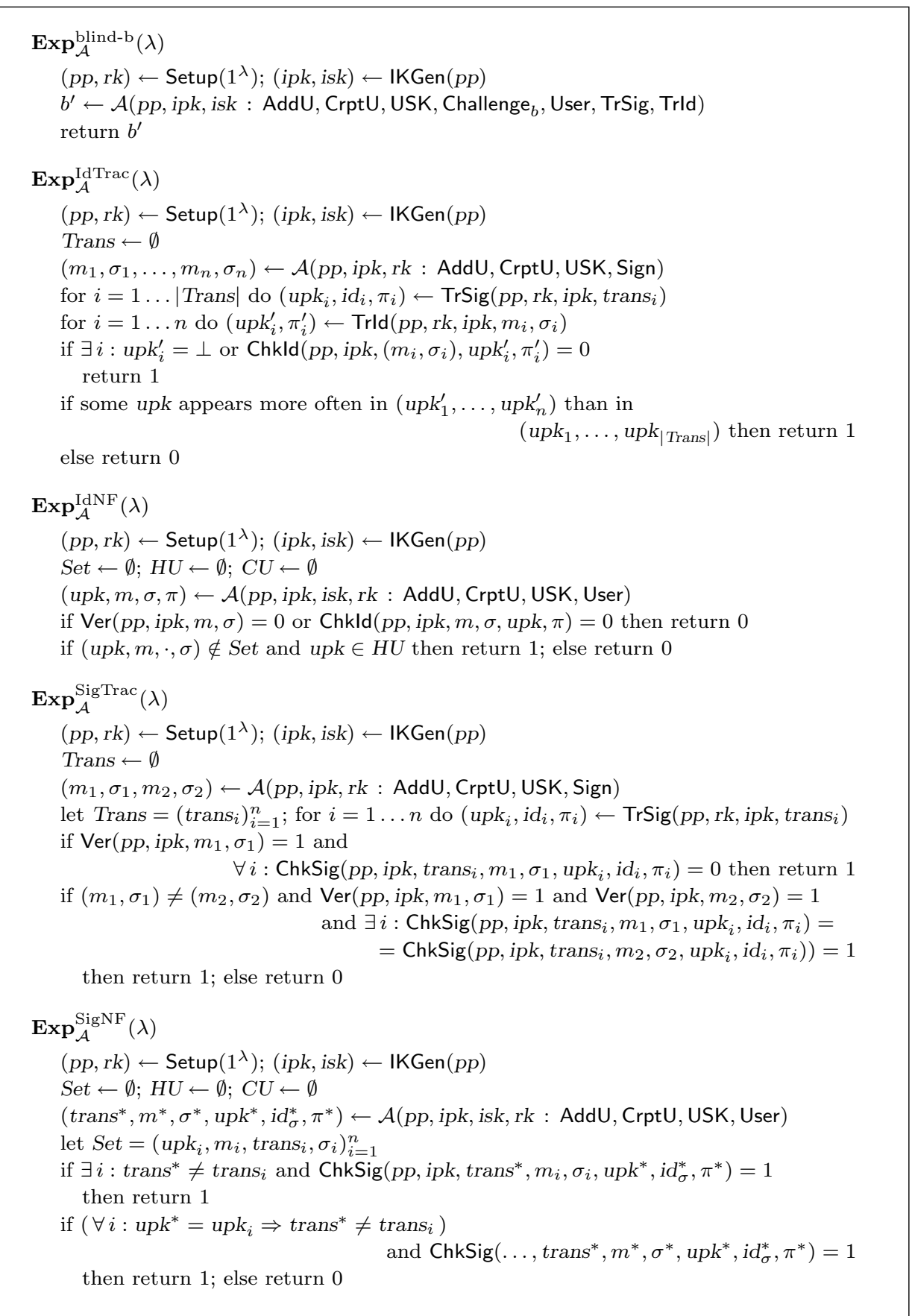

Fig. 1. Security experiments for fair blind signatures 
We propose the following experiment leading to a stronger notion: the adversary gets the authority's key and impersonates corrupt users, who, via the Sign oracle can request signatures from the honest issuer. The latter is simulated by the experiment and keeps a set Trans of transcripts of oracle calls. Eventually, the adversary outputs a set of message/signature pairs. The experiment opens all transcripts to get a list of users to which signatures were issued. Another list of users is constructed by opening the returned signatures. The adversary wins if there exists a user who appears more often in the second list than in the first, or if $\perp$ is in the second list or if any of the proofs output by the opening algorithm do not verify. Note that the notion of [HT07] is implied by ours.

Non-Frameability Notions. Non-frameability means that not even a coalition of everyone else can "frame" an honest user. For example, no adversary can output a signature which opens to a user who did not participate in its issuing. In [HT07], the adversary outputs a message/signature pair, which is then opened by the experiment to determine if it "framed" a user. Analogously to [BSZ05] (who defined non-frameability for group signatures), we define a stronger notion requiring the adversary to output an incriminating signature, an honest user and a valid proof, that the signature opens to that user. Note that only this formalization makes the $\pi$ output by the tracing algorithms a proof, as it guarantees that no adversary can produce a proof that verifies for a false opening.

IDENTITY Non-FrameABILITY. In [HT07], the adversary wins if it produces a pair $(m, \sigma)$ such that, when opened to upk, we have $(m, \sigma, u p k) \notin S e t$. This seems to guarantee a strong notion of unforgeability where an adversary modifying a signature wins the game. This is however not the case in the scheme proposed in [HT07]: the final signature is a proof of knowledge of some values computed by the issuer made non-interactive by the Fiat-Shamir heuristic; hence from a given signature issuing session the user may derive several valid signatures on a message $m$. For that reason, the model in [HT07] considers that two signatures are different only if the underlying secrets are different. We adopt the same convention in this paper in that we consider two signatures equivalent if they have the same identifier.

Signature Non-Frameability. Non-frameability of signature tracing intuitively means: even if everyone else colludes against an honest user, they cannot produce a transcript that opens to an honest signature. In the definition proposed in [HT07], the adversary plays the issuer in that he gets his secret key. However, he has no possibility to communicate with honest users since the challenger plays the issuer in the signature issuing sessions with honest users and the adversary only gets the transcripts. His goal is to produce a new message/signature pair (one that does not emanate from a User-oracle call) such that an honest transcript opens to it.

We give the following security notion which we think is more intuitive. No corrupt issuer can produce a transcript of an issuing session and one of the following: either a public key of an honest user and a proof that this user participated in the transcript whereas he did not; or a signature identifier of an honest signature coming from a different session and a proof that the transcript 
opens to it. Similarly to signatures we consider two transcripts equivalent if the contain the same user randomness and the same issuer randomness.

Unforgeability. Consider an adversary that breaks the classical security notion for blind signatures, one-more unforgeability, i.e., after $q-1$ Sign-oracle queries, he outputs $q$ signatures on different messages. We show that the adversary must have broken signature traceability: indeed since there are more signatures than transcripts, either there is a signature which no transcripts points to, or there is a transcript that points to two signatures.

\section{Assumptions}

A (symmetric) bilinear group is a tuple $\left(p, \mathbb{G}, \mathbb{G}_{T}, e, G\right)$ where $(\mathbb{G}, \cdot)$ and $\left(\mathbb{G}_{T}, \cdot\right)$ are two cyclic groups of prime order $p, G$ is a generator of $\mathbb{G}$, and $e: \mathbb{G} \times \mathbb{G} \rightarrow$ $\mathbb{G}_{T}$ is a non-degenerate bilinear map, i.e., $\forall U, V \in \mathbb{G} \forall a, b \in \mathbb{Z}: e\left(U^{a}, V^{b}\right)=$ $e(U, V)^{a b}$, and $e(G, G)$ is a generator of $\mathbb{G}_{T}$.

The Decision Linear (DLIN) Assumption [BBS04], in $\left(p, \mathbb{G}, \mathbb{G}_{T}, e, G\right)$ states that given $\left(G^{\alpha}, G^{\beta}, G^{r \alpha}, G^{s \beta}, G^{t}\right)$ for random $\alpha, \beta, r, s \in \mathbb{Z}_{p}$, it is hard to decide whether $t=r+s$ or $t$ is random.

The following two assumptions were introduced by [FPV09] and [Fuc09], respectively. Under the knowledge of exponent assumption [Dam92], the first is equivalent to $\mathrm{SDH}[\mathrm{BB} 04]$ and the second is equivalent to computing discrete logarithms.

Assumption 1 ( $q$-DHSDH). Given $\left(G, H, K, X=G^{x}\right) \in \mathbb{G}^{4}$ and $q-1$ tuples

$$
\left(A_{i}=\left(K G^{v_{i}}\right)^{\frac{1}{x+d_{i}}}, C_{i}=G^{d_{i}}, D_{i}=H^{d_{i}}, V_{i}=G^{v_{i}}, W_{i}=H^{v_{i}}\right)_{i=1}^{q-1},
$$

for $d_{i}, v_{i} \leftarrow \mathbb{Z}_{p}$, it is hard to output a new tuple $(A, C, D, V, W)$ that satisfies

$$
e(A, X C)=e(K V, G) \quad e(C, H)=e(G, D) \quad e(V, H)=e(G, W)
$$

The next assumption states that, given $(G, H, T) \in \mathbb{G}^{3}$, it is hard to produce a non-trivial $\left(G^{m}, H^{m}, G^{r}, H^{r}\right)$ such that $G^{m}=T^{r}$.

Assumption 2 (HDL). Given a random triple $(G, H, T) \in \mathbb{G}^{3}$, it is hard to output $(M, N, R, S) \neq(1,1,1,1)$ such that

$$
e(R, T)=e(M, G) \quad e(M, H)=e(G, N) \quad e(R, H)=e(G, S)
$$

\section{Tools}

We recall some tools from the literature which we use to construct our scheme. 


\subsection{A Signature Scheme to Sign Group Elements}

We present the signature scheme from [Fuc09], which is secure against chosenmessage attacks under Assumptions 1 and 2. Its message space is the set of Diffie-Hellman pairs $\mathcal{D} \mathcal{H}:=\left\{(A, B) \in \mathbb{G}^{2} \mid \exists \alpha: A=G^{\alpha}, B=H^{\alpha}\right\}$ w.r.t. two fixed generators $G, H \in \mathbb{G}$. Note that $(A, B) \in \mathcal{D} \mathcal{H}$ iff $e(A, H)=e(G, B)$.

\section{Scheme $1\left(\operatorname{Sig}_{1}\right)$.}

Setup $_{1}$ Given $\left(p, \mathbb{G}, \mathbb{G}_{T}, e, G\right)$, choose additional generators $H, K, T \in \mathbb{G}$.

KeyGen $_{1}$ Choose $s k=x \leftarrow \mathbb{Z}_{p}$ and set $v k=G^{x}$.

$\operatorname{Sign}_{1}$ A signature on $(M, N) \in \mathcal{D H}$ under public key $G^{x}$, is defined as

$$
\left(S_{1}:=\left(K T^{r} M\right)^{\frac{1}{x+d}}, S_{2}:=G^{d}, S_{3}:=H^{d}, S_{4}:=G^{r}, S_{5}:=H^{r}\right),
$$

for random $d, r \leftarrow \mathbb{Z}_{p}$

Verify $_{1}\left(S_{1}, S_{2}, S_{3}, S_{4}, S_{5}\right)$ is valid on $(M, N) \in \mathcal{D H}$ under public key $v k=X$ iff

$$
\begin{array}{ll}
e\left(S_{1}, X S_{2}\right)=e(K M, G) e\left(T, S_{4}\right) & e\left(S_{2}, H\right)=e\left(G, S_{3}\right) \\
e\left(S_{4}, H\right)=e\left(G, S_{5}\right)
\end{array}
$$

\subsection{Groth-Sahai Proofs}

We sketch the results of Groth and Sahai [GS08] on proofs of satisfiability of sets of equations over a bilinear group $\left(p, \mathbb{G}, \mathbb{G}_{T}, e, G\right)$. Due to the complexity of their methodology, we merely give what is needed for our results and refer to the full version of [GS08] for any additional details.

We define a key for linear commitments. Choose $\alpha, \beta, r_{1}, r_{2} \in \mathbb{Z}_{p}$ and define $U=G^{\alpha}, V=G^{\beta}$, and $\mathbf{u}_{1}:=(U, 0, G), \mathbf{u}_{2}:=(0, V, G), \mathbf{u}_{3}:=\left(W_{1}, W_{2}, W_{3}\right)$ where $W_{1}:=U^{r_{1}}, W_{2}:=V^{r_{2}}$, for random $r_{1}, r_{2} \leftarrow \mathbb{Z}_{p}$, and $W_{3}$ is either

- soundness setting: $W_{3}:=G^{r_{1}+r_{2}}$ (which makes $\overrightarrow{\mathbf{u}}$ a binding key)

- witness-indistinguishable setting: $W_{3}:=G^{r_{1}+r_{2}-1}$ (making $\overrightarrow{\mathbf{u}}$ a hiding key)

Under key $c k=\left(U, V, W_{1}, W_{2}, W_{3}\right)$, a commitment to a group element $X \in \mathbb{G}$ using randomness $\left(s_{1}, s_{2}, s_{3}\right) \leftarrow \mathbb{Z}_{p}^{3}$ is defined as (with $\iota(X):=(0,0, X)$ )

$$
\begin{aligned}
\operatorname{Com}\left(c k, X ;\left(s_{1}, s_{2}, s_{3}\right)\right) & :=\iota(X) \cdot \prod_{i=1}^{3} \mathbf{u}_{i}^{s_{i}} \\
= & \left(U^{s_{1}} W_{1}^{s_{3}}, V^{s_{2}} W_{2}^{s_{3}}, X G^{s_{1}+s_{2}} W_{3}^{s_{3}}\right) .
\end{aligned}
$$

In the soundness setting, given the extraction key ek $:=(\alpha, \beta)$, the committed value can be extracted from a commitment $\mathbf{c}=\left(c_{1}, c_{2}, c_{3}\right)$. On the other hand, in the witness-indistinguishable (WI) setting, $\mathbf{c}$ is equally distributed for every $X$. The two settings are indistinguishable under the DLIN assumption.

A pairing-product equation is an equation for variables $\mathcal{Y}_{1}, \ldots, \mathcal{Y}_{n} \in \mathbb{G}$ of the form

$$
\prod_{i=1}^{n} e\left(\mathcal{A}_{i}, \mathcal{Y}_{i}\right) \prod_{i=1}^{n} \prod_{j=1}^{n} e\left(\mathcal{Y}_{i}, \mathcal{Y}_{j}\right)^{\gamma_{i, j}}=t_{T}
$$


with $\mathcal{A}_{i} \in \mathbb{G}, \gamma_{i, j} \in \mathbb{Z}_{p}$ and $t_{T} \in \mathbb{G}_{T}$.

To show satisfiability of a set of equations of this form, one first makes commitments to a satisfying witness (i.e., an assignment to the variables of each equation) and then adds a "proof" per equation. Groth and Sahai describe how to construct these: they are in $\mathbb{G}^{3 \times 3}$. In the soundness setting, if the proof is valid, then Extr extracts the witness satisfying the pairing-product equation. In the WI setting, commitments and proofs of different witnesses which both satisfy the same pairing-product equation are equally distributed.

\subsection{Commit and Encrypt}

In order to build CCA-anonymous group signatures, Groth [Gro07] uses the following technique: a group signature consists of linear commitments to a certified signature and Groth-Sahai proofs that the committed values constitute a valid signature. CPA-anonymity follows from WI of GS proofs: once the commitment key has been replaced by a perfectly hiding one, a group signature reveals no information about the signer. However, in order to simulate opening queries in the WI setting, some commitments are doubled with a tag-based encryption under Kiltz' scheme [Kil06] and a Groth-Sahai NIZK proof that the committed and the encrypted value are the same. To produce a group signature, the user first chooses a key pair for a one-time signature scheme, uses the verification key as the tag for the encryption and the secret key to sign the group signature.

By $\operatorname{Sig}_{\text {ot }}=\left(K\right.$ eyGen ${ }_{\text {ot }}, \operatorname{Sign}_{\mathrm{ot}}$, Ver $\left._{\mathrm{ot}}\right)$ we will denote the signature scheme discussed in $\S 5.2$ which satisfies the required security notion. By CEP (commitencrypt-prove) we denote the following:

$$
\begin{aligned}
& \operatorname{CEP}(c k, p k, t a g, m s g ;(\rho, r)):= \\
& \quad(\operatorname{Com}(c k, m s g ; \rho), \operatorname{Enc}(p k, t a g, m s g ; r), \operatorname{NizkEq}(c k, p k, m s g, t a g, \rho, r))
\end{aligned}
$$

where Enc denotes Kiltz' encryption and NizkEq denotes a Groth-Sahai NIZK proof that the commitment and the encryption contain the same plaintext (cf. [Gro07]). We say that an output $\psi=(\mathbf{c}, C, \zeta)$ of CEP is valid if the ciphertext and the zero-knowledge proof are valid.

\section{New Tools}

\subsection{A Scheme To Sign Three Diffie-Hellman Pairs}

We extend the scheme from $\S 4.1$, so it signs three messages at once; we prove existential unforgeability against adversaries making a particular chosen message attack: the first message is given (as usual) as a Diffie-Hellman pair, whereas the second and third message are queried as their logarithms, i.e., instead of querying $\left(G^{v}, H^{v}\right)$, the adversary has to give $v$ explicitly. As we will see, this combines smoothly with our application. 
Scheme $2\left(\operatorname{Sig}_{3}\right)$.

$\operatorname{Setup}_{3}(\mathcal{G})$ Given $\mathcal{G}=\left(p, \mathbb{G}, \mathbb{G}_{T}, e, G\right)$, choose additional generators $H, K, T \in \mathbb{G}$.

$\operatorname{KeyGen}_{3}(\mathcal{G})$ Choose $s k=(x, \ell, u) \leftarrow \mathbb{Z}_{p}^{3}$ and set $v k=\left(G^{x}, G^{\ell}, G^{u}\right)$.

$\operatorname{Sign}_{3}((x, \ell, u),(M, N, Y, Z, V, W))$ A signature on $((M, N),(Y, Z),(V, W)) \in \mathcal{D H}^{3}$ under public key $G^{x}$, is defined as (for random $d, r \leftarrow \mathbb{Z}_{p}$ )

$$
\left(S_{1}:=\left(K T^{r} M Y^{\ell} V^{u}\right)^{\frac{1}{x+d}}, S_{2}:=G^{d}, S_{3}:=H^{d}, S_{4}:=G^{r}, S_{5}:=H^{r}\right)
$$

Verify $_{3}\left(S_{1}, S_{2}, S_{3}, S_{4}, S_{5}\right)$ is valid on messages $(M, N),(Y, Z),(V, W)$ under a public key $(X, L, U)$ iff

$$
e\left(S_{1}, X S_{2}\right)=e(K M, G) e\left(T, S_{4}\right) e(L, Y) e(U, V) \quad \begin{aligned}
& e\left(S_{2}, H\right)=e\left(G, S_{3}\right) \\
& e\left(S_{4}, H\right)=e\left(G, S_{5}\right)
\end{aligned}
$$

Theorem 1. $\mathrm{Sig}_{3}$ is existentially unforgeable against adversaries making chosen message attacks of the form $\left(\left(M_{1}, N_{1}\right), m_{2}, m_{3}\right)$.

Proof. Let $\left(M_{i}, N_{i}, y_{i}, v_{i}\right)$ be the queries, $\left(A_{i}, C_{i}, D_{i}, R_{i}=G^{r_{i}}, S_{i}\right)$ be the responses. Let $(M, N, Y, Z, V, W)$ and $\left(A, C, D, R=G^{r}, S\right)$ be a successful forgery. We distinguish 4 types of forgers (where $Y_{i}:=G^{y_{i}}, V_{i}:=G^{v_{i}}$ ):

Type I

$$
\forall i: T^{r_{i}} M_{i} Y_{i}^{\ell} V_{i}^{u} \neq T^{r} M Y^{\ell} V^{u}
$$

Type II $\quad \exists i: T^{r_{i}} M_{i} Y_{i}^{\ell} V_{i}^{u}=T^{r} M Y^{\ell} V^{u} \wedge M_{i} Y_{i}^{\ell} V_{i}^{u} \neq M Y^{\ell} V^{u}$

Type III $\quad \exists i: M_{i} Y_{i}^{\ell} V_{i}^{u}=M Y^{\ell} V^{u} \wedge M_{i} V_{i}^{u} \neq M V^{u}$

Type IV

$$
\exists i: M_{i} Y_{i}^{\ell} V_{i}^{u}=M Y^{\ell} V^{u} \wedge M_{i} V_{i}^{u}=M V^{u}
$$

Type $\mathbf{I}$ is reduced to DHSDH. Let $\left(G, H, K,\left(A_{i}, C_{i}, D_{i}, E_{i}, F_{i}\right)_{i=1}^{q-1}\right)$ be an instance. Choose and $t, \ell, u \leftarrow \mathbb{Z}_{p}$ and set $T=G^{t}, L=G^{\ell}$ and $U=G^{u}$. A signature on $\left(M_{i}, N_{i}, Y_{i}, Z_{i}, y_{i}, V_{i}, W_{i}, v_{i}\right)$ is (after a consistency check) answered as $\left(A_{i}, C_{i}, D_{i},\left(E_{i} M_{i}^{-1} Y_{i}^{-\ell} V_{i}^{-u}\right)^{1 / t},\left(F_{i} N_{i}^{-1} Z_{i}^{-\ell} W_{i}^{-u}\right)^{1 / t}\right)$. After a successful forgery, return $\left(A, C, D, R^{t} M Y^{\ell} V^{u}, S^{t} N Z^{\ell} W^{u}\right)$, which is a valid DHSDH solution by (5).

Type II is reduced to HDL. Let $(G, H, T)$ be an HDL instance. Generate the rest of the parameters and a public key and answer the queries by signing. After a successful forgery, return

$$
\left(M Y^{\ell} V^{u} M_{i}^{-1} Y_{i}^{-\ell} V_{i}^{-u}, N Z^{\ell} W^{u} N_{i}^{-1} Z_{i}^{-\ell} W_{i}^{-u}, R_{i} R^{-1}, S_{i} S^{-1}\right),
$$

which is non-trivial by (6).

Type III is reduced to HDL. Let $(G, H, L)$ be an instance. Choose $K, T \leftarrow \mathbb{G}$ and $x, u \leftarrow \mathbb{Z}_{p}$ and return the parameters and public key $\left(X=G^{x}, L, U=\right.$ $\left.G^{u}\right)$. Thanks to the $y_{i}$ in the signing queries, we can simulate them: return $\left(\left(K T^{r_{i}} M_{i} L^{y_{i}} V_{i}^{u}\right)^{\frac{1}{x+d_{i}}}, G^{d_{i}}, H^{d_{i}}, G^{r_{i}}, H^{r_{i}}\right)$. From (7) we have $M V^{u} M_{i}^{-1} V_{i}^{-u}=$ $Y_{i}^{\ell} Y^{-\ell}=L^{y_{i}-y}$, so from a successful forgery, we can return

$$
\left(M V^{u} M_{i}^{-1} V_{i}^{-u}, N W^{u} N_{i}^{-1} W_{i}^{-u}, Y_{i} Y^{-1}, Z_{i} Z^{-1}\right)
$$

which is non-trivial by (7). 
Type IV is also reduced to HDL. Let $(G, H, U)$ be an HDL instance. Choose $K, T \leftarrow \mathbb{G}$ and $x, \ell \leftarrow \mathbb{Z}_{p}$ and return the parameters and public key $(X=$ $\left.G^{x}, L=G^{\ell}, U\right)$. Thanks to the $v_{i}$ in the signing queries, we can simulate them: return $\left(\left(K T^{r_{i}} M_{i} Y_{i}^{\ell} U^{v_{i}}\right)^{\frac{1}{x+d_{i}}}, G^{d_{i}}, H^{d_{i}}, G^{r_{i}}, H^{r_{i}}\right)$. From a successful forgery of Type IV we have $M M_{i}^{-1}=U^{v_{i}-v}$ from (7), we can thus return $\left(M M_{i}^{-1}, N N_{i}^{-1}, V_{i} V^{-1}, W_{i} W^{-1}\right)$, which is non-trivial, $(M, N, Y, Z, V, W)$ being a valid forgery and $(Y, Z)=\left(Y_{i}, Z_{i}\right)$ by $(8)$.

\subsection{A Simulation-Sound Non-Interactive Zero-Knowledge Proof of Knowledge of a CDH Solution}

Let $(G, F, V)$ be elements of $\mathbb{G}$. We construct a simulation-sound non-interactive zero-knowledge (SSNIZK) proof of knowledge (PoK) of $W$ s.t. $e(V, F)=e(G, W)$. We follow the overall approach by Groth [Gro06]. The common reference string (CRS) contains a CRS for Groth-Sahai (GS) proofs and a public key for a EUFCMA signature scheme Sig. A proof is done as follows: choose a key pair for a one-time signature scheme $\mathbf{S i g}_{\mathrm{ot}}$, and make a witness-indistinguishable GS proof of the following: either to know $W$, a $\mathrm{CDH}$ solution for $(G, F, V)$ or to know a signature on the chosen one-time key which is valid under the public key from the $\mathrm{CRS} ;{ }^{2}$ finally sign the proof using the one-time key. A SSNIZKPoK is verified by checking the GS proofs and the one-time signature. Knowing the signing key corresponding to the key in the CRS, one can simulate proofs by using as a witness a signature on the one-time key.

We require that a proof consist of group elements only and is verified by checking a set of pairing-product equations. This can be achieved by using the scheme from Scheme 1 and a one-time scheme to sign group elements using the commitment scheme in [Gro09] based on the DLIN assumption. ${ }^{3}$

\section{A Fair Blind Signature Scheme}

The basis of our protocol is the blind automorphic signature scheme from [Fuc09]: the user randomizes the message to be signed, the issuer produces a pre-signature from which the user obtains a signature by removing the randomness; the final signature is a Groth-Sahai proof of knowledge of the resulting signature.

\footnotetext{
${ }^{2}$ In [Gro06] it is shown how to express a disjunction of two equation sets by a new set of equations.

3 The strong one-time signature scheme used in [Gro06] works as follows: The verification key is a Pedersen commitment to 0 . To sign a message, using the trapdoor, the commitment is opened to the message. By putting a second trapdoor in the commitment scheme, we can simulate one signing query and use a forger to break the binding property of the commitment scheme. In [Gro09], Groth proposes a scheme to commit to group elements. Using his scheme rather than Pedersen commitments, we can construct an efficient one-time signature scheme for group elements whose signatures consist of group elements (see Appendix A).
} 
In our scheme, in addition to the message, the issuer signs the user's public key, and an identifier of the signature, which the issuer and the user define jointly. Note that the issuer may neither learn the user's public key nor the identifier. To guarantee provable tracings, the user signs what she sends in the issuing protocol and the final signature. To prevent malicious issuers from producing a transcript that opens to an honest signature, the proof contains a SSNIZK proof of knowledge of the randomness introduced by the user. To guarantee blindness against adversaries with tracing oracles, the elements that serve as proofs of correct tracing are additionally encrypted and the transcript (and final signature) is signed with a one-time key (cf. $\S$ 4.3).

To trace a signature, the authority extracts tracing information from the commitments as well as signatures that act as proofs.

\subsection{Setup and Key Generation}

Setup. Choose a bilinear group $\mathcal{G}:=\left(p, \mathbb{G}, \mathbb{G}_{T}, e, G\right)$ and parameters $(H, K, T)$ for $\mathbf{S i g}_{3}$. Pick $F, H^{\prime} \leftarrow \mathbb{G}$, a commitment and extraction key (ck, ek) for GrothSahai proofs, a key pair for tag-based encryption (epk, esk) and sscrs, a common reference string for SSNIZKPoK.

Output $p p:=\left(\mathcal{G}, G, H, K, T, F, H^{\prime}, c k, e p k\right.$, sscrs $)$ and $r k:=e k$.

Key Generation. Both IKGen and UKGen is defined as KeyGen, i.e., the key generation algorithm for $\mathbf{S i g}_{1}$ (and $\mathbf{S i g}_{3}$ ).

\subsection{The Signature Issuing Protocol and Verification}

The common inputs are ( $\left.p p, i p k=G^{x}\right)$, the issuer's additional input is isk $=x$, the user's additional inputs are $\left(u p k=G^{y}, u s k=y,(M, N) \in \mathcal{D} \mathcal{H}\right)$.

1. User Choose $\eta, v^{\prime} \leftarrow \mathbb{Z}_{p}$ and set $P=G^{\eta}, Q=F^{\eta}, V^{\prime}=G^{v^{\prime}}, W^{\prime}=F^{v^{\prime}}$.

Produce $\xi \leftarrow \operatorname{SSNIZKPoK}\left(\operatorname{sscrs},\left(P, V^{\prime}\right),\left(Q, W^{\prime}\right)\right) .{ }^{4}$

Choose $\left(v k_{\mathrm{ot}}^{\prime}, s k_{\mathrm{ot}}^{\prime}\right) \leftarrow \operatorname{KeyGen}_{\mathrm{ot}}(\mathcal{G})$ and set $\Sigma^{\prime} \leftarrow \operatorname{Sign}\left(u s k, v k_{\mathrm{ot}}^{\prime}\right) .^{5}$

Send the following

(a) $Y=G^{y}, Z=H^{y}, v k_{\mathrm{ot}}^{\prime}, \Sigma^{\prime}$,

(b) $\mathbf{c}_{M}=\operatorname{Com}(c k, M) ; \mathbf{c}_{N}:=\operatorname{Com}(c k, N)$,

$\psi_{P}, \psi_{V}, \vec{\psi}_{\xi}$, with $\psi_{\odot}:=\operatorname{CEP}\left(c k, e p k, v k_{\mathrm{ot}}^{\prime}, \odot\right)$,

a proof $\phi_{M}$ that $(M, N) \in \mathcal{D H}$ and a proof $\phi_{\xi}$ of validity of $\xi$,

(c) $J:=\left(K M L^{y} U^{v^{\prime}}\right)^{\frac{1}{\eta}}$,

(d) a zero-knowledge proof $\zeta$ of knowledge of $\eta, y$ and $v^{\prime}$ such that $-Y=G^{y}$

${ }^{4}$ A simulation-sound non-interactive proof of knowledge of $Q$ and $W^{\prime}$ such that $e\left(V^{\prime}, F\right)=e\left(G, W^{\prime}\right)$ and $e(P, F)=e(G, Q)$. (cf. $\left.\S 5.2\right)$.

${ }^{5}$ The message space for Sig is the set of DH pairs w.r.t. $\left(G, H^{\prime}\right)$. Since all logarithms of $v k_{\mathrm{ot}}$ are known when picking a key, the user can complete the second components of the DH pairs. 
- $\mathbf{c}_{V}$ commits to $G^{v^{\prime}} ;$ and

- $\mathbf{c}_{M}$ commits to $J^{\eta} L^{-y} U^{-v^{\prime}} K^{-1}$.

(e) $\operatorname{sig}^{\prime} \leftarrow \operatorname{Sign}_{\mathrm{ot}}\left(\operatorname{sk}_{\mathrm{ot}}^{\prime},\left(Y, Z, \Sigma^{\prime}, \mathbf{c}_{M}, \mathbf{c}_{N}, \psi_{P}, \psi_{V}, \vec{\psi}_{\xi}, \phi_{M}, \phi_{\xi}, J, \zeta, v k_{\mathrm{ot}}^{\prime}\right)\right)$.

2. Issuer If $\Sigma^{\prime}, \psi_{P}, \psi_{V}, \vec{\psi}_{\xi}$ as well as $\phi_{M}, \phi_{\xi}$, sig' and the proof of knowledge are valid, choose $d, r, v^{\prime \prime} \leftarrow \mathbb{Z}_{p}$ and send:

$$
A^{\prime}:=\left(J T^{r} U^{v^{\prime \prime}}\right)^{\frac{1}{x+d}} \quad C:=G^{d} \quad D:=F^{d} \quad R^{\prime}:=G^{r} \quad S^{\prime}:=H^{r} \quad v^{\prime \prime}
$$

The user does the following:

(a) set $A:=\left(A^{\prime}\right)^{\eta}, R:=\left(R^{\prime}\right)^{\eta}, S:=\left(S^{\prime}\right)^{\eta}, V:=G^{v^{\prime}+\eta v^{\prime \prime}}, W:=H^{v^{\prime}+\eta v^{\prime \prime}}$ and check whether $(A, C, D, R, S)$ is valid on $((M, N),(Y, Z),(V, W))$ under ipk;

(b) choose $\left(v k_{\mathrm{ot}}, s k_{\mathrm{ot}}\right) \leftarrow \operatorname{KeyGen}_{\mathrm{ot}}$ and define $\Sigma=\operatorname{Sign}\left(y, v k_{\mathrm{ot}}\right)$;

(c) make commitments $\mathbf{c}_{A}, \mathbf{c}_{C}, \mathbf{c}_{D}, \mathbf{c}_{R}, \mathbf{c}_{S}$ to $A, C, D, R, S$ under ck;

(d) run $\operatorname{CEP}\left(c k, e p k, v k_{\mathrm{ot}}, \cdot\right)$ on $Y, Z$ and $\Sigma$ (let $\psi_{Y}, \psi_{Z}$ and $\vec{\psi}_{\Sigma}$ denote the outputs);

(e) make a proof $\phi_{Y}$ that $(Y, Z) \in \mathcal{D H}$ and proofs $\phi_{S}$ and $\phi_{\Sigma}$ of validity of the signatures $(A, C, D, R, S)$ and $\Sigma$;

(f) set sig $\leftarrow \operatorname{Sign}_{\text {ot }}\left(\operatorname{sk}_{\mathrm{ot}},\left(V, W, M, N, \mathbf{c}_{A}, \mathbf{c}_{C}, \mathbf{c}_{D}, \mathbf{c}_{R}, \mathbf{c}_{S}\right.\right.$,

$$
\left.\left.\psi_{Y}, \psi_{Z}, \vec{\psi}_{\Sigma}, \phi_{Y}, \phi_{S}, \phi_{\Sigma}, v k_{\mathrm{ot}}\right)\right) \text {. }
$$

The signature on $(M, N)$ is

$$
\left(V, W, \mathbf{c}_{A}, \mathbf{c}_{C}, \mathbf{c}_{D}, \mathbf{c}_{R}, \mathbf{c}_{S}, \psi_{Y}, \psi_{Z}, \vec{\psi}_{\Sigma}, \phi_{Y}, \phi_{S}, \phi_{\Sigma}, v k_{\mathrm{ot}}, \operatorname{sig}\right)
$$

A signature is verified by verifying sig under $v k_{\mathrm{ot}}$, checking the proofs $\phi_{Y}, \phi_{S}$ and $\phi_{\Sigma}$, and verifying the encryptions and NIZK proofs in $\psi_{Y}, \psi_{Z}$ and $\vec{\psi}_{\Sigma}$.

Remark 1. As mentioned by [Fuc09], there are two possible instantiations of the zero-knowledge proof of knowledge in 1.d: either using bit-by-bit techniques (which does not increase the rounds of the protocol); or optimizing the amount of data sent by adding 3 rounds using interactive concurrent Schnorr proofs.

Theorem 2. The above scheme is an unforgeable blind signature (in the classical sense) under the DLIN, the DHSDH and the HDL assumptions.

The proof of unforgeability is by reduction to unforgeability of Scheme 2, analogously to the proof in [Fuc09]. Note that by additionally extracting $y$ and $v^{\prime}$ from the proof of knowledge, the simulator can make the special signing queries. The proof of blindness is also analogous to [Fuc09]. 


\subsection{Tracing Algorithms}

Opening of a Transcript ("Signature Tracing"). Given a transcript

$$
\left(Y, Z, \Sigma^{\prime}, \mathbf{c}_{M}, \mathbf{c}_{N}, \psi_{P}, \psi_{V}, \vec{\psi}_{\xi}, \phi_{M}, \phi_{\xi}, J, \zeta, v k_{\mathrm{ot}}^{\prime}, \operatorname{sig}^{\prime}\right), \quad v^{\prime \prime}
$$

verify $\Sigma^{\prime}, s i g^{\prime}$, the proofs $\phi_{M}$ and $\phi_{\xi}$ and the ciphertexts and proofs in $\psi_{P}, \psi_{V}$ and $\vec{\psi}_{\xi}$. If everything is valid, use rk $=e k$ to open the commitments in $\psi_{P}, \psi_{V}$ and $\vec{\psi}_{\xi}$ to $P, V^{\prime}$ and $\xi$ respectively and set $V:=V^{\prime} P^{v^{\prime \prime}}=G^{v^{\prime}+\eta v^{\prime \prime}}$.

Return $i d_{\sigma}:=V$, upk $=Y$ and $\pi:=\left(V^{\prime}, P, v^{\prime \prime}, \xi, \Sigma^{\prime}\right)$. The proof $\pi$ is verified by checking $V=V^{\prime} P^{v^{\prime \prime}}$, verifying $\xi$ on $V^{\prime}$ and $P$, and verifying $\Sigma^{\prime}$ under $Y$.

Opening of a Signature ("Identity Tracing"). Given a valid signature

$$
\left(V, W, \mathbf{c}_{A}, \mathbf{c}_{C}, \mathbf{c}_{D}, \mathbf{c}_{R}, \mathbf{c}_{S}, \psi_{Y}, \psi_{Z}, \vec{\psi}_{\Sigma}, \phi_{Y}, \phi_{S}, \phi_{\Sigma}, v k_{\mathrm{ot}}, \operatorname{sig}\right),
$$

open the commitments in $\psi_{Y}, \psi_{Z}$ and $\vec{\psi}_{\Sigma}$ using ek and return upk $=Y$ and $\pi=\Sigma$. A proof $\pi$ is verified by checking if $\Sigma$ is a valid signature on $(V, W)$ under $Y$.

\section{Security Proofs}

Theorem 3. The above scheme is a secure fair blind signature scheme (in the model defined in § 2) under the DLIN, the DHSDH and the HDL assumptions.

Due to space limitation, we sketch the security proofs of all security notions.

Blindness (under DLIN). In the witness-indistinguishability setting of GrothSahai proofs, the commitments and proofs do not reveal anything - and neither do the ciphertexts. Furthermore, for every $M$ and $V$, there exist $\eta$ and $v^{\prime}$ that explain $J$. In more detail: the proof proceeds by games, Game 0 being the original game. In Game 1, we use the decryption key for the tag-based encryptions to answer queries to trace signatures and identities. The zero-knowledge proofs in the $\psi$ 's guarantees that the committed and the encrypted values are the same; the games are thus indistinguishable.

In Game 2, we replace the commitment key ck by a witness indistinguishable one. In Game 3, we simulate the NIZK proofs in the $\psi$ 's and in Game 4, we replace the ciphertexts in the $\psi$ 's by encryptions of 0 . Games 3 and 4 are indistinguishable by selective-tag weak CCA security of Kiltz' cryptosystem (which follows from DLIN): by unforgeability of the one-time signature, the adversary cannot query a different transcript (or signature) with the same tag as the target transcript (or signature), therefore we can answer all tracing queries.

In Game 5, we simulate the zero-knowledge proofs in Step 1d. In this game, the adversary's view is the following: $J=\left(K M L^{y} U^{v^{\prime}}\right)^{\frac{1}{\eta}}$ and $M^{*}, V^{*}$ which are either $M$ and $G^{v^{\prime}+\eta v^{\prime \prime}}$ or not. Let small letters denote the logarithms of the respective capital letters. Then for every $m^{*}=\log M^{*}, v^{*}=\log V^{*}$ there exist 
$\eta, v^{\prime}$ such that $v^{*}=v^{\prime}+\eta v^{\prime \prime}$ and $j=\frac{1}{\eta}\left(k+m^{*}+y l+v^{\prime} u\right)$, i.e., that make $M^{*}, V^{*}$ consistent with $J$. In Game 5, which is indistinguishable from the original game, the adversary has thus no information on whether a given transcript corresponds to a given signature.

Identity Traceability (under DHSDH+HDL). An adversary wins if he can produce a set of valid pairs $\left(m_{i}, \sigma_{i}\right)$ s.t. either $(\mathrm{I})$ for one of them the tracing returns $\perp$ or the proof does not verify, or (II) a user appears more often in the openings of the signatures than in the openings of the transcripts. By soundness of Groth-Sahai, we can always extract a user public key and a valid signature. If an adversary wins by (II), then we can use him to forge a $\mathbf{S i g}_{3}$ signature:

Given parameters and a public key for $\mathbf{S i g}_{3}$, we set up the rest of the parameters for the blind signature. Whenever the adversary queries his Sign oracle, we do the following: use ek to extract $(M, N)$ from $\left(\mathbf{c}_{M}, \mathbf{c}_{N}\right)$, extract $\eta, y$ and $v^{\prime}$ from the zero-knowledge proof $\zeta$. Choose $v^{\prime \prime} \leftarrow \mathbb{Z}_{p}$ and query $\left(M, N, y, v^{\prime}+\eta v^{\prime \prime}\right)$ to signing oracle to receive $(A, C, D, R, S)$. Return $\left(A^{\frac{1}{\eta}}, C, D, R^{\frac{1}{\eta}}, S^{\frac{1}{\eta}}, v^{\prime \prime}\right)$. If the adversary wins by outputting a set of different (i.e., with distinct identifiers $(V, W))$ blind signatures with one user appearing more often then in the transcripts, then among the $\mathbf{S i g}_{3}$ signatures extracted from the blind signatures there must be a forgery.

Identity Non-Frameability (under DLIN+DHSDH+HDL). Using a successful adversary, we can either forge a signature by the user on $v k_{\text {ot }}^{\prime}$ or a one-time signature (which is secure under DLIN). More precisely, we call an adversary of Type I if it reuses a one-time key from the signatures it received from the User oracle. Since the signature $\mathcal{A}$ returns must not be contained in Set, it is different from the one containing the reused one-time key. The contained one-time signature can thus be returned as a forgery.

An adversary of Type II uses a new one-time key for the returned signature. We use $\mathcal{A}$ to forge a Sig signature. The simulator is given parameters $\left(H^{\prime}, K, T\right)$ and a public key $Y$ for Sig, sets it as one of the honest users' upk and queries its signing oracle to simulate the user. Having set $H=G^{h}$, the simulator can produce $Z=H^{y}=Y^{h}$ in the User oracle queries. Since the $v k_{\text {ot }}^{\prime}$ contained $\mathcal{A}$ 's output was never queried, we get a valid forgery.

Signature Traceability (under DHSDH+HDL). If the adversary wins by outputting a message/signature pair with an identifier $(V, W)$ s.t. no transcript opens to it, we can extract a $\mathbf{S i g}_{3}$ signature on $(M, N, Y, Z, V, W)$ without having ever queried a signature on any $(\cdot, \cdot, \cdot, \cdot, V, W)$. The simulation is done analogously to the proof of identity traceability. Consider an adversary outputting two message/signature pairs with two different messages. With overwhelming probability, the identifiers of the signatures are different (since $v^{\prime \prime}$ is chosen randomly by the experiment after the adversary chose $v^{\prime}$ and $\eta$ ). Thus the simulator only asked one query for $(\cdot, \cdot, \cdot, \cdot, V, W)$. The second signature can therefore be returned as a forgery by the simulator. Lastly, if the messages are the same, the signatures must be different, thus have different identifiers. One of the ChkSig calls in the experiment returns thus 0 . 
Signature Non-Frameability (under DLIN+DHSDH+HDL). There are two ways for an issuer to "wrongfully" open a transcript: either he opens it to a user (not necessarily honest) and an identifier of a signature which was produced by an honest user in another session; or it opens it to an honest user who has not participated in the issuing session.

Framing AN HONEST SignATURE. Suppose the adversary impersonating the issuer manages to produce a new opening of a transcript that leads to an honestly generated signature.

We reduce this framing attack to break $\mathrm{CDH}$, whose hardness is implied by that of DLIN. Let $\left(G, F, V^{\prime}\right)$ be a CDH challenge, i.e., we seek to produce $W^{\prime}:=F^{\left(\log _{G} V^{\prime}\right)}$. Set up the parameters of the scheme setting $H=G^{h}$ and knowing the trapdoor for SSNIZKPoK. In one call of the adversary's User oracle calls we do the following: choose $\eta \leftarrow \mathbb{Z}_{p}$ and use $V^{\prime}$ from the CDH challenge. Simulate the proof of knowledge of $W^{\prime}$. Let $v^{\prime \prime}$ be the value returned from the adversary, and $\left(V:=V^{\prime} P^{\eta}, W:=V^{h}\right)$ be the identifier of the resulting signature.

Suppose the adversary produces a proof $\left(\bar{V}^{\prime}, \bar{P}, \bar{v}^{\prime \prime}, \bar{\pi}, \bar{\Sigma}\right)$ with $\left(\bar{V}^{\prime}, \bar{P}\right) \neq$ $\left(V^{\prime}, P\right)$ for the honest identifier $(V, W)$. By simulation soundness of SSNIZKPoK, we can extract $\bar{W}^{\prime}=F^{\left(\log _{G} \bar{V}^{\prime}\right)}$ and $\bar{Q}=F^{\left(\log _{G} \bar{P}\right)}$. From $V^{\prime} G^{\eta v^{\prime \prime}}=V=\bar{V}^{\prime} \bar{P}^{\bar{v}^{\prime \prime}}$ we get $V^{\prime}=\bar{V}^{\prime} \bar{P}^{\bar{v}^{\prime \prime}} G^{-\eta v^{\prime \prime}}$ and thus $W^{\prime}=\bar{W}^{\prime} \bar{Q}^{\bar{v}^{\prime \prime}} F^{-\eta v^{\prime \prime}}$ is a CDH solution. If the adversary recycles $\left(V^{\prime}, P\right)$, then it must find a new $v^{\prime \prime}$ which leads to a $V$ of an honest signature, and thus has to solve a discrete logarithm.

FrAming AN HONEST USER. Suppose the adversary outputs an opening of a transcript and a proof revealing an honest user that has never participated in that transcript. Analogously to the proof for signature traceability, we can use the adversary to either forge a signature under a user public key or to forge a one-time signature.

\section{Conclusion}

We presented the first efficient fair blind signature scheme with a security proof in the standard model. The scheme satisfies a new security model that strengthens the one proposed by Hufschmitt and Traoré in 2007. The new scheme is efficient (both keys and signatures consist of a constant number of group elements) and does not rely on any new assumptions. As byproducts, we proposed an extension of Fuchsbauer's automorphic signatures and a simulation-sound non-interactive zero-knowledge proof of knowledge of a Diffie-Hellman solution, both compatible with the Groth-Sahai methodology.

\section{Acknowledgments}

This work was supported by the French ANR 07-TCOM-013-04 PACE Project, the European Commission through the IST Program under Contract ICT-2007216646 ECRYPT II, and EADS. 


\section{References}

[AF96] Masayuki Abe and Eiichiro Fujisaki. How to date blind signatures. In Kwangjo Kim and Tsutomu Matsumoto, editors, ASIACRYPT'96, volume 1163 of $L N C S$, pages 244-251. Springer, November 1996.

[AO01] Masayuki Abe and Miyako Ohkubo. Provably secure fair blind signatures with tight revocation. In Colin Boyd, editor, ASIACRYPT 2001, volume 2248 of $L N C S$, pages 583-602. Springer, December 2001.

[BB04] Dan Boneh and Xavier Boyen. Short signatures without random oracles. In Christian Cachin and Jan Camenisch, editors, EUROCRYPT 2004, volume 3027 of LNCS, pages 56-73. Springer, May 2004.

[BBS04] Dan Boneh, Xavier Boyen, and Hovav Shacham. Short group signatures. In Matthew Franklin, editor, CRYPTO 2004, volume 3152 of LNCS, pages 41-55. Springer, August 2004.

[BSZ05] Mihir Bellare, Haixia Shi, and Chong Zhang. Foundations of group signatures: The case of dynamic groups. In Alfred Menezes, editor, $C T$ RSA 2005, volume 3376 of LNCS, pages 136-153. Springer, February 2005.

[CGT06] Sébastien Canard, Matthieu Gaud, and Jacques Traoré. Defeating malicious servers in a blind signatures based voting system. In Giovanni Di Crescenzo and Avi Rubin, editors, FC 2006, volume 4107 of LNCS, pages 148-153. Springer, February / March 2006.

[Cha83] David Chaum. Blind signatures for untraceable payments. In David Chaum, Ronald L. Rivest, and Alan T. Sherman, editors, CRYPTO'82, pages 199203. Plenum Press, New York, USA, 1983.

[Dam92] Ivan Damgård. Towards practical public key systems secure against chosen ciphertext attacks. In Joan Feigenbaum, editor, CRYPTO'91, volume 576 of LNCS, pages 445-456. Springer, August 1992.

[FPV09] Georg Fuchsbauer, David Pointcheval, and Damien Vergnaud. Transferable anonymous constant-size fair e-cash. In CANS 2009: 8th International Conference on Cryptology And Network Security, 2009. (to appear) Preliminary version available at http://eprint.iacr.org/2009/146.

[Fuc09] Georg Fuchsbauer. Automorphic signatures in bilinear groups. Cryptology ePrint Archive, Report 2009/320, 2009. http://eprint.iacr.org/.

[GL07] Jens Groth and Steve Lu. A non-interactive shuffle with pairing based verifiability. In Kaoru Kurosawa, editor, ASIACRYPT 2007, volume 4833 of $L N C S$, pages 51-67. Springer, December 2007.

[Gro06] Jens Groth. Simulation-sound NIZK proofs for a practical language and constant size group signatures. In Xuejia Lai and Kefei Chen, editors, ASI$A C R Y P T$ 2006, volume 4284 of $L N C S$, pages 444-459. Springer, December 2006.

[Gro07] Jens Groth. Fully anonymous group signatures without random oracles. In Kaoru Kurosawa, editor, ASIACRYPT 200\%, volume 4833 of LNCS, pages 164-180. Springer, December 2007.

[Gro09] Jens Groth. Homomorphic trapdoor commitments to group elements. Cryptology ePrint Archive, Report 2009/007, 2009. http://eprint.iacr.org/.

[GS08] Jens Groth and Amit Sahai. Efficient non-interactive proof systems for bilinear groups. In Nigel P. Smart, editor, EUROCRYPT 2008, volume 4965 of $L N C S$, pages 415-432. Springer, April 2008.

[GT03] Matthieu Gaud and Jacques Traoré. On the anonymity of fair offline e-cash systems. In Rebecca Wright, editor, FC 2003, volume 2742 of $L N C S$, pages 34-50. Springer, January 2003. 
[HT07] Emeline Hufschmitt and Jacques Traoré. Fair blind signatures revisited. In Tsuyoshi Takagi, Tatsuaki Okamoto, Eiji Okamoto, and Takeshi Okamoto, editors, PAIRING 200\%, volume 4575 of LNCS, pages 268-292. Springer, July 2007.

[Kil06] Eike Kiltz. Chosen-ciphertext security from tag-based encryption. In Shai Halevi and Tal Rabin, editors, TCC 2006, volume 3876 of LNCS, pages 581-600. Springer, March 2006.

[Nao03] Moni Naor. On cryptographic assumptions and challenges (invited talk). In Dan Boneh, editor, CRYPTO 2003, volume 2729 of LNCS, pages 96-109. Springer, August 2003.

[Oka06] Tatsuaki Okamoto. Efficient blind and partially blind signatures without random oracles. In Shai Halevi and Tal Rabin, editors, TCC 2006, volume 3876 of $L N C S$, pages 80-99. Springer, March 2006.

$\left[\mathrm{OMA}^{+} 99\right]$ Miyako Ohkubo, Fumiaki Miura, Masayuki Abe, Atsushi Fujioka, and Tatsuaki Okamoto. An improvement on a practical secret voting scheme. In Masahiro Mambo and Yuliang Zheng, editors, ISW'99, volume 1729 of LNCS, pages 225-234. Springer, November 1999.

[RS10] Markus Rückert and Dominique Schröder. Fair partially blind signatures. to appear at AFRICACRYPT '10, 2010.

[SPC95] Markus Stadler, Jean-Marc Piveteau, and Jan Camenisch. Fair blind signatures. In Louis C. Guillou and Jean-Jacques Quisquater, editors, EUROCRYPT'95, volume 921 of LNCS, pages 209-219. Springer, May 1995. 


\section{A A One-Time Signature on Vectors of Group Elements}

Our one-time signature is based on the simultaneous triple pairing assumption (STP) stating that the following problem is hard:

Given random generators $\left(g_{r}, h_{r}, g_{s}, h_{s}, g_{t}, h_{t}\right) \in \mathbb{G}^{6}$, output $(r, s, t) \in$ $\mathbb{G}^{3} \backslash\{(1,1,1)\}$ such that

$$
e\left(g_{r}, r\right) e\left(g_{s}, s\right) e\left(g_{t}, t\right)=1 \quad e\left(h_{r}, r\right) e\left(h_{s}, s\right) e\left(h_{t}, t\right)=1
$$

In Groth [Gro09] proves that DLIN implies STP and presents a homomorphic commitment scheme whose binding property is implied by the above assumption. We transform his commitment scheme to a one-time signature scheme analogous to the scheme in [Gro06] based on Pedersen commitments. The signature uses a commitment with an additional trapdoor. The public key is a commitment to 0 and a signature is a trapdoor opening of the commitment to the message.

We give a scheme with message space $\mathbb{G}^{n}$.

KeyGen ${ }_{\text {ot }}$ Choose $x_{r}, y_{r}, x_{s}, y_{s}, x_{t}, y_{t}, x_{1}, y_{1}, \ldots, x_{n}, y_{n}, v, w \leftarrow \mathbb{Z}_{p}$ such that $x_{r} y_{s} \neq$

$x_{s} y_{r}$. Define $g_{i}:=g^{x_{i}}, h_{i}=: g^{y_{i}}$ for $i=r, s, t, 1, \ldots, n, c=g^{v}, d=g^{w}$. Let

$\alpha, \beta, \gamma, \delta$ s.t. $\left(\begin{array}{ll}\alpha & \beta \\ \gamma & \delta\end{array}\right)=\left(\begin{array}{ll}x_{r} & x_{s} \\ y_{r} & y_{s}\end{array}\right)^{-1}$. The public key is

$$
\left(c, d, \vec{g}=\left(g_{r}, g_{s}, g_{t}, g_{1}, \ldots, g_{n}\right), \vec{h}=\left(h_{r}, h_{s}, h_{t}, h_{1}, \ldots, h_{n}\right)\right)
$$

and the secret key is $\left(\alpha, \beta, \gamma, \delta, x_{t}, y_{t}, x_{1}, y_{1}, \ldots, x_{n}, y_{n}\right)$.

Sign $_{\text {ot }}$ To sign a message $\left(m_{1}, \ldots, m_{n}\right) \in \mathbb{G}^{n}$. Choose $t \leftarrow \mathbb{G}$ and set $a:=$

$c t^{-x_{t}} \prod m_{i}^{-x_{i}}$ and $b:=d t^{-y_{t}} \prod m_{i}^{-y_{i}}$. Return $\left(r=a^{\alpha} b^{\beta}, s=a^{\gamma} b^{\delta}, t\right)$.

Verify $_{\text {ot }}$ A signature $(r, s, t)$ is verified on $\left(m_{1}, \ldots, m_{n}\right)$ by checking

$$
\begin{aligned}
e\left(g_{r}, r\right) e\left(g_{s}, s\right) e\left(g_{t}, t\right) \prod e\left(g_{i}, m_{i}\right) & =e(c, g) \\
e\left(h_{r}, r\right) e\left(h_{s}, s\right) e\left(h_{t}, t\right) \prod e\left(h_{i}, m_{i}\right) & =e(d, g)
\end{aligned}
$$

A signature produced by $\operatorname{Sign}_{\text {ot }}$ is indeed accepted by Verify ${ }_{\text {ot }}$ since:

$$
\begin{aligned}
e\left(g_{r}, r\right) e\left(g_{s}, s\right) e\left(g_{t}, t\right) \prod e\left(g_{i}, m_{i}\right) & =e\left(g_{r}, a^{\alpha} b^{\beta}\right) e\left(g_{s}, a^{\gamma} b^{\delta}\right) e\left(g_{t}, t\right) \prod e\left(g_{i}, m_{i}\right) \\
& =e\left(a^{\alpha x_{r}+\gamma x_{s}}, g\right) e\left(b^{\beta x_{r}+\delta x_{s}}, g\right) e\left(g_{t}, t\right) \prod e\left(g_{i}, m_{i}\right) \\
& =e(a, g) e\left(g_{t}, t\right) \prod e\left(g_{i}, m_{i}\right) \\
& =e\left(c t^{-x_{t}} \prod m_{i}^{-x_{i}}, g\right) e\left(g_{t}, t\right) \prod e\left(g_{i}, m_{i}\right) \\
& =e(c, g) e\left(t^{-x_{t}}, g\right) e\left(g_{t}, t\right) \prod e\left(m_{i}^{-x_{i}}, g\right) e\left(g_{i}, m_{i}\right) \\
& =e(c, g)
\end{aligned}
$$

and similarly

$$
\begin{aligned}
e\left(h_{r}, r\right) e\left(h_{s}, s\right) e\left(h_{t}, t\right) \prod e\left(h_{i}, m_{i}\right) & =e\left(h_{r}, a^{\alpha} b^{\beta}\right) e\left(h_{s}, a^{\gamma} b^{\delta}\right) e\left(h_{t}, t\right) \prod e\left(h_{i}, m_{i}\right) \\
& =e\left(a^{\alpha y_{r}+\gamma y_{s}}, g\right) e\left(b^{\beta y_{r}+\delta y_{s}}, g\right) e\left(h_{t}, t\right) \prod e\left(h_{i}, m_{i}\right) \\
& =e(b, g) e\left(h_{t}, t\right) \prod e\left(h_{i}, m_{i}\right) \\
& =e(d, g)
\end{aligned}
$$


Assuming STP, the signature is strongly unforgeable under a one-time chosen message attack.

Let $\left(g_{r}, h_{r}, g_{s}, h_{s}, g_{t}, h_{t}\right)$ be an STP instance. If $\left(g_{r}, g_{s}, h_{r}, h_{s}\right)$ is a Diffie-Hellman (DH) tuple, (i.e., $\left.e\left(g_{r}, h_{s}\right)=e\left(g_{s}, h_{r}\right)\right)$, we have an STP solution $\left(g_{s}, g_{r}^{-1}, 1\right)$, since $e\left(g_{r}, g_{s}\right) e\left(g_{s}, g_{r}^{-1}\right) e\left(g_{t}, 1\right)=1$ and $e\left(h_{r}, g_{s}\right) e\left(h_{s}, g_{r}^{-1}\right) e\left(h_{t}, 1\right)=1$.

If $\left(g_{r}, g_{s}, h_{r}, h_{s}\right)$ is not a DH-tuple, we choose $\bar{\rho}, \bar{\sigma}, \bar{\tau}, \rho_{1}, \sigma_{1}, \tau_{1}, \ldots, \rho_{n}, \sigma_{n}, \tau_{n} \leftarrow$ $\mathbb{Z}_{p}$ and set $g_{i}:=g_{r}^{\rho_{i}} g_{s}^{\sigma_{i}} g_{t}^{\tau_{i}}, h_{i}:=h_{r}^{\rho_{i}} h_{s}^{\sigma_{i}} h_{t}^{\tau_{i}}$, for $1 \leq i \leq n$; and $c:=g_{r}^{\bar{\rho}} g_{s}^{\bar{\sigma}} g_{t}^{\bar{\tau}}, d:=$ $h_{r}^{\bar{\rho}} h_{s}^{\bar{\sigma}} h_{t}^{\bar{\tau}}$. Since $\left(g_{r}, g_{s}\right)$ and $\left(h_{r}, h_{s}\right)$ are "linearly independent", all these group elements look random. We give the adversary the public key $(c, d, \vec{g}, \vec{h})$. The signing query for $\left(m_{1}, \ldots, m_{n}\right)$ is answered by returning $r=g^{\bar{\rho}} \prod m_{i}^{-\rho_{i}}, s=g^{\bar{\sigma}} \prod m_{i}^{-\sigma_{i}}$, $t=g^{\bar{\tau}} \prod m_{i}^{-\tau_{i}}$. We have:

$$
\begin{aligned}
e\left(g_{r}, r\right) e\left(g_{s}, s\right) e\left(g_{t}, t\right) & =e\left(g_{r}, g^{\bar{\rho}} \prod m_{i}^{-\rho_{i}}\right) e\left(g_{s}, g^{\bar{\sigma}} \prod m_{i}^{-\sigma_{i}}\right) e\left(g_{t}, g^{\bar{\tau}} \prod m_{i}^{-\tau_{i}}\right) \\
& =e\left(g_{r}^{\bar{\rho}} g_{s}^{\bar{\sigma}} g_{t}^{\bar{\tau}}, g\right) \prod\left(g_{r}^{-\rho_{i}} g_{s}^{-\sigma_{i}} g_{t}^{-\tau_{i}}, m_{i}\right) \\
& =e(c, g) \prod e\left(g_{i}^{-1}, m_{i}\right)
\end{aligned}
$$

and similarly

$$
e\left(h_{r}, r\right) e\left(h_{s}, s\right) e\left(h_{t}, t\right)=e(d, g) \prod e\left(h_{i}^{-1}, m_{i}\right) .
$$

Thus $(r, s, t)$ is a valid signature for $\left(m_{1}, \ldots, m_{n}\right)$ and since $\bar{\tau}$ and the $\tau_{i}$ 's are perfectly hidden, this looks like a random signature produced by $\operatorname{Sign}_{\mathrm{ot}}$.

Suppose the adversary outputs $\left(m_{1}^{\prime}, \ldots, m_{n}^{\prime}, r^{\prime}, s^{\prime}, t^{\prime}\right) \neq\left(m_{1}, \ldots, m_{n}, r, s, t\right)$. Dividing the verification relation for each signatures yields:

$$
\begin{aligned}
e\left(g_{r}, r^{\prime} r^{-1} \prod\left(m_{i}^{\prime} m_{i}^{-1}\right)^{\rho_{i}}\right) e\left(g_{s}, s^{\prime} s^{-1} \prod\left(m_{i}^{\prime} m_{i}^{-1}\right)^{\sigma_{i}}\right) e\left(g_{t}, t^{\prime} t^{-1} \prod\left(m_{i}^{\prime} m_{i}^{-1}\right)^{\tau_{i}}\right) & =1 \\
e\left(h_{r}, r^{\prime} r^{-1} \prod\left(m_{i}^{\prime} m_{i}^{-1}\right)^{\rho_{i}}\right) e\left(h_{s}, s^{\prime} s^{-1} \prod\left(m_{i}^{\prime} m_{i}^{-1}\right)^{\sigma_{i}}\right) e\left(h_{t}, t^{\prime} t^{-1} \prod\left(m_{i}^{\prime} m_{i}^{-1}\right)^{\tau_{i}}\right) & =1
\end{aligned}
$$

If $\left(m_{1}^{\prime}, \ldots, m_{n}^{\prime}\right)=\left(m_{1}, \ldots, m_{n}\right)$, then $\left(r^{\prime} r^{-1}, s^{\prime} s^{-1}, t^{\prime} t^{-1}\right) \neq(1,1,1)$ and these relations provide a solution to the STP problem. Otherwise, if we denote $I \subset$ $\{1, \ldots, n\}$, the set of indices for which $m_{i}^{\prime} \neq m_{i}$ and $n_{i}:=m_{i}^{\prime} m_{i}^{-1}$, the probability that the adversary's output satisfies $r^{\prime} \prod_{i \in S} n_{i}^{\rho_{i}}=r$ is upper-bounded by $1 / p$ since the $\rho_{i}$ 's are perfectly hidden. Therefore if $\left(m_{1}^{\prime}, \ldots, m_{n}^{\prime}\right) \neq\left(m_{1}, \ldots, m_{n}\right)$, we also obtain a solution to the STP problem with overwhelming probability. 